\title{
Combination versus monotherapy for nosocomial pneumonia
}

\author{
H. Lode
}

ABSTRACT: Combination antibiotic therapy of nosocomial pneumonia is sometimes appropriate and desirable; however, it should be used judiciously. When pneumonia appears in the first 5 days following hospital admission and in the absence of other risk factors for infection by multidrug-resistant pathogens, the infection is likely to be due to a pathogen acquired in the community and is likely to be sensitive to most antibiotics.

These infections should generally be treated with monotherapy. However, pathogens resistant to multiple drugs are increasingly common in the hospital and intensive care unit setting. In the presence of risk factors for such pathogens, any single drug may prove ineffective; treatment with two or more drugs theoretically increases the likelihood that the pathogen will be sensitive to at least one of them. However, combination therapy also increases the cost and the likelihood of adverse effects, as well as the possibility of drug interactions, if the two are not chosen wisely.

The crucial question is whether combination antibiotic therapy actually improves clinical outcome. Most clinical trials suggest that monotherapy and combination therapy provide equivalent efficacy. However, these studies have uniformly excluded the most seriously ill patients: those with Acute Physiology and Chronic Evaluation-II scores $>20$.

It is concluded that available studies provide no concrete evidence to support the use of combination therapy in moderately ill patients and provide no data for the treatment of seriously ill patients who might be most likely to benefit.

KEYWORDS: Antibiotics, combination therapy, monotherapy, resistance, ventilator-associated pneumonia

$T$ he treatment of nosocomial pneumonia, particularly ventilator-associated pneumonia (VAP), is becoming increasingly difficult. Not only are the pathogens encountered in the intensive care unit (ICU) more likely to be antibiotic-resistant, but the frequency of multidrug resistance is also increasing. The likelihood that the pathogen will be resistant to any given drug constitutes a strong argument for the administration of two or more drugs in the hope that one will prove effective. However, combination therapy has its own drawbacks, so it must be employed judiciously.

\section{GROWING FREQUENCY OF RESISTANCE}

Pulmonary pathogens encountered in the ICU fall into two general groups. In the first group are the pathogens generally sensitive to the most common antibiotics. These include Streptococcus pneumoniae, Haemophilus influenzae, methicillinsensitive Staphylococcus aureus (MSSA) and anaerobes generally. In the second group are organisms that are inherently difficult to treat, and/or likely to be resistant to multiple drugs. These include Pseudomonas aeruginosa, methicillin-resistant S. aureus (MRSA), Acinetobacter spp. and bacteria that have acquired the ability to produce extendedspectrum $\beta$-lactamases (ESBLs), not only Klebsiella but Escherichia coli and others. Mixed infections are common in both groups.

Table 1 shows that the frequency with which varies from country to country [1]. Resistance to oxacillin or ciprofloxacin in Italy has been reported to be almost $60 \%$ for $S$. aureus, compared with $\sim 50 \%$ in the USA. Close to $30 \%$ of Klebisella pneumoniae isolates in Italy were shown to produce ESBLs and were resistant to ceftazidime. In Germany, the resistance of E. coli to ciprofloxacin has risen from 12.4 to $20 \%$. Consequently, ciprofloxacin is no longer indicated for the empiric treatment of nosocomial pneumonia.

In many countries, $P$. aeruginosa, a very problematic pathogen, is resistant to a range of antibiotics [2]. Ciprofloxacin was once the most active fluoroquinolone against this organism, but figure 1 shows that its resistance is currently near these bacteria are resistant to specific antibiotics
AFFILIATIONS

${ }^{*}$ Research Centre for Medical

Studies, Institute for Clinical

Pharmacology, Charité-

Universitätsmedizin, Berlin, Germany.

CORRESPONDENCE

H. Lode

Research Centre for Medical Studies Institute for Clinical Pharmacology

Charité-Universitätsmedizin

Hohenzollerndamm 2

D-10717 Berlin

Germany

Fax: 493088719386

E-mail: haloheck@zedat.fu-berlin.de

\section{STATEMENT OF INTEREST}

$H$. Lode has received grants from and is a speaker for Bayer AG, SanofiArentis, Janssen-Cilag, Pfizer, Wyeth and Novartis 
TABLE 1 Resistance rates among bacterial pathogens in intensive care units, 2000-2002

\begin{tabular}{|c|c|c|c|c|c|c|}
\hline Staphylococcus aureus & Oxacillin & 52.3 & 19.7 & 59.4 & 21.0 & 40.6 \\
\hline Klebsiella pneumoniae & Ceftazidime & 10.1 & 2.2 & 28.5 & 8.2 & 5.2 \\
\hline \multirow[t]{2}{*}{ Escherichia coli } & Piperacillin/tazobactam & 3.6 & 2.4 & 2.4 & 3.4 & 1.1 \\
\hline & Ciprofloxacin & 10.7 & 9.5 & 12.7 & 12.4 & 6.5 \\
\hline \multirow{2}{*}{ Pseudomonas aeruginosa } & Imipenem & 22.1 & 18.2 & 27.8 & 19.0 & 21.4 \\
\hline & Ciprofloxacin & 33.1 & 30.2 & 38.8 & 24.4 & 40.6 \\
\hline
\end{tabular}

Data are presented as \%. Reproduced from [1] with permission from the publisher.

$30 \%$ in both the USA and France and nearer $40 \%$ in Italy. If there are risk factors for $P$. aeruginosa infection, ciprofloxacin should not be used for empiric monotherapy.

An alternative to ineffective monotherapy is combination therapy. A combination of antibiotics provides a broader spectrum of coverage than any single antibiotic alone. A wellchosen combination should be synergistic and provide an antibacterial spectrum greater than the sum of their individual activities. Combination therapy may reduce the likelihood that resistance will emerge during therapy, since only bacteria with mutations providing resistance to both antibiotics will be able to survive and grow [3].

While these points argue for combination therapy, there are also weaknesses to the argument. For example, monotherapy is less costly and there are likely to be fewer adverse effects. Occasionally, rather than synergism, antagonism can occur with the wrong combination therapy. Furthermore, the metabolism of one drug can interfere with the metabolism of the other, requiring dose adjustments that may not always be clear. Therefore, the primary issue is whether combination therapy is more efficacious than monotherapy.

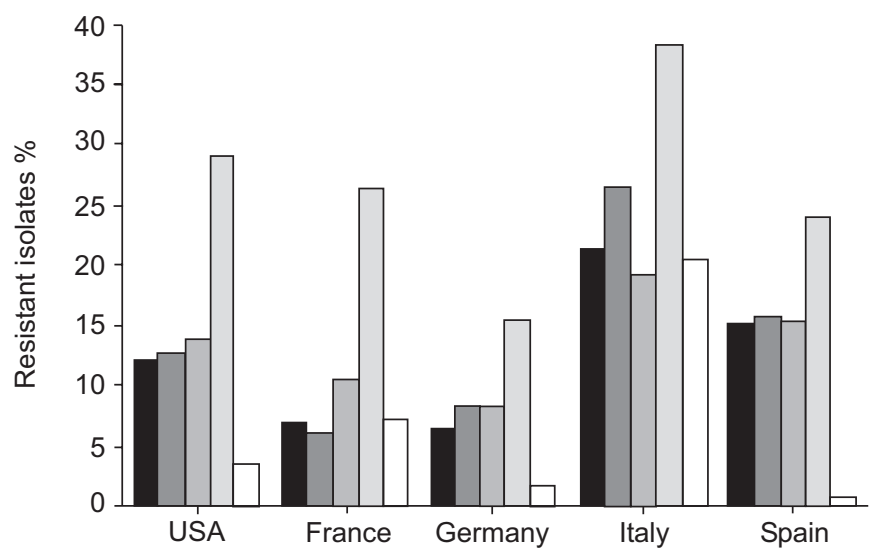

FIGURE 1. Profile of Pseudomonas aeruginosa resistance by country and antibiotic. $\mathbf{\square}$ : piperacillin/tazobactam; $\mathbf{\square}$ : ceftazidime; $\mathbf{\square}$ : imipenem; $\mathbf{\square}$ : ciprofloxacin; $\square$ : amikacin. Reproduced from [2] with permission from the publisher.

\section{DOES COMBINATION THERAPY LIMIT THE EMERGENCE OF RESISTANCE?}

When $P$. aeruginosa was cultured in vitro in the presence of either ceftazidime alone or ceftazidime in combination with tobramycin (fig. 2), ceftazidime monotherapy led to a steady increase in the minimum inhibitory concentration with time that was not seen with the combination of ceftazidime and tobramycin. In this case, combination therapy largely abolished emergent resistance [4]. Other studies using animal models also showed combination therapy to inhibit emergent resistance, principally in $P$. aeruginosa. However, there are no strong clinical trials to show that combination therapy inhibits emergent resistance [4].

\section{MONOTHERAPY VERSUS COMBINATION THERAPY: COMPARISONS OF EFFICACY}

In a study comparing clinical outcomes and inflammatory parameters of cefepime monotherapy with two combination therapies, 74 patients with clinical signs of VAP were recruited of these, 59 had microbiologically confirmed VAP [5]. A group of 20 patients were randomly assigned to cefepime monotherapy, 19 to cefepime with amikacin and 20 to cefepime with

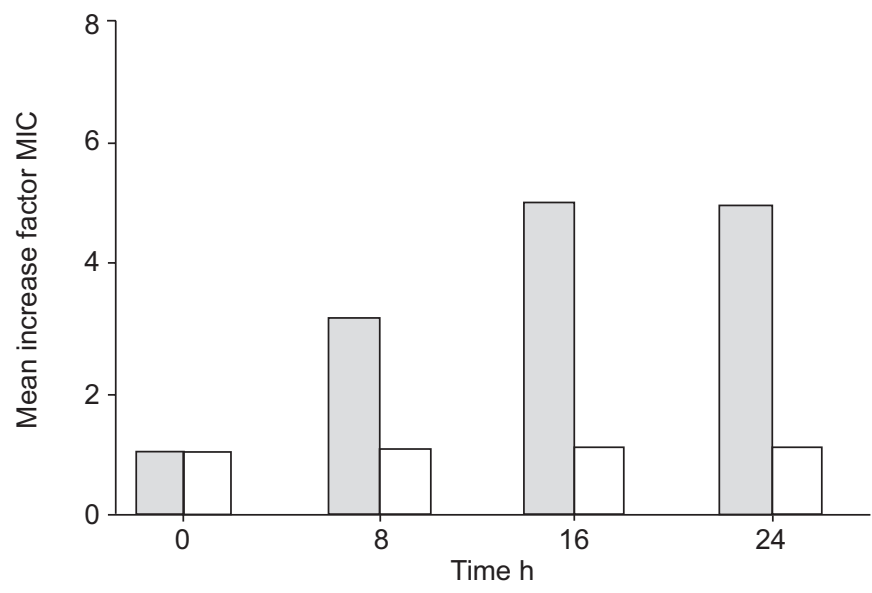

FIGURE 2. Mean change (log) in ceftazidime mean inhibitory concentration (MIC) against Pseudomonas aeruginosa in the presence $(\square)$ and absence ( $\square$ ) of concomitant tobramycin in vitro. Reproduced from [4] with permission from the publisher. 

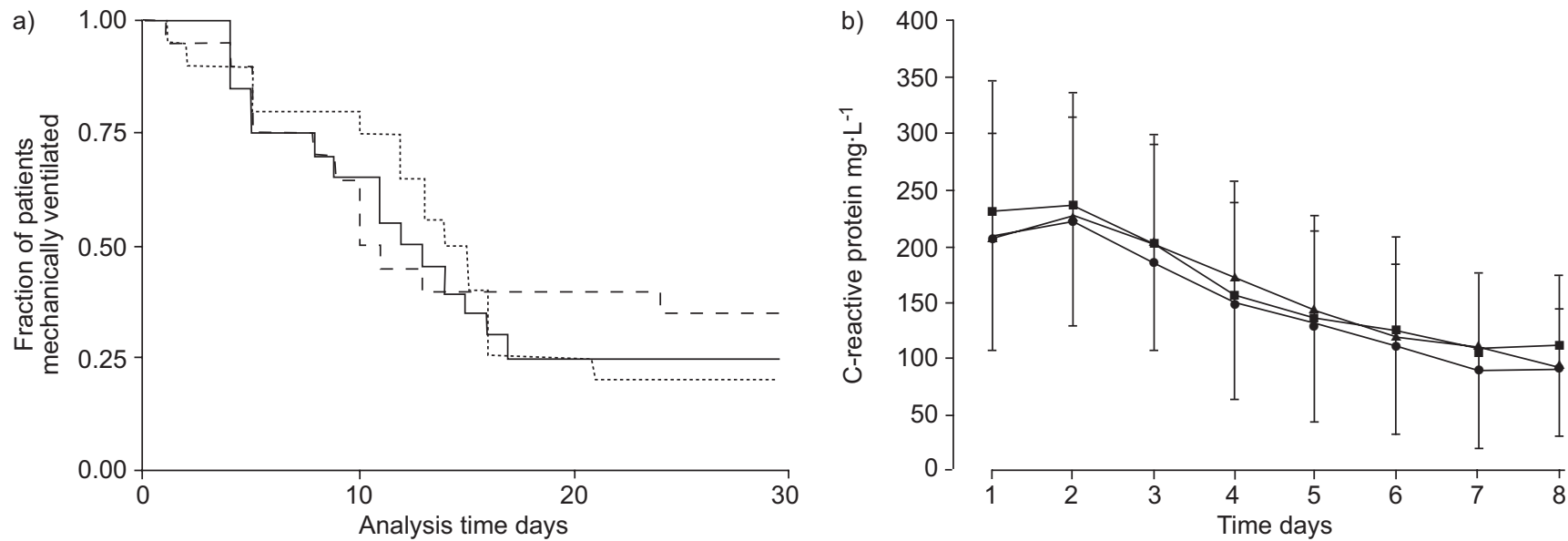

FIGURE 3. a) Kaplan-Meier curves for the duration of mechanical ventilation under cefepime monotherapy ( $\longrightarrow$ ) and two combination therapies: cefepime with levofloxacin $(---)$ and cefepime with amikacin $(\cdots \cdots \cdot)$. p $=0.74$. b) Time-course of C-reactive protein levels under cefepime monotherapy $(\bullet)$ and cefepime in combination with levofloxacin $(\mathbf{\Lambda})$ or amikacin ( $\mathbf{\square})$. Reproduced from [5] with permission from the publisher.

levofloxacin. The median length of stay in the ICU following diagnosis was similar for all three groups (15, 16 and 14 days, respectively; $\mathrm{p}=$ nonsignificant). Figure 3 illustrates that the respective duration of mechanical ventilation and the timecourse of C-reactive protein levels were similar. Measures of the arterial oxygen tension/fraction of inspired oxygen ratio, temperature and leukocytosis did not differ between the groups [5]. One patient on cefepime monotherapy, with a $P$. aeruginosa infection following cardiac surgery, died of sepsis. The other nine deaths were divided among the three groups and were not clearly attributable to infection [5]. It was concluded that combination therapy using a fourth-generation cephalosporin with either an aminoglycoside or a fluoroquinolone was not associated with a clinical or biological benefit when compared with cephalosporin monotherapy for common, susceptible pathogens causing VAP [5]. Of the many comparative studies for the treatment of VAP, six are summarised in table 2 [6-11]. Perhaps the best of these studies compared imipenem alone with the combination of imipenem and netilmicin [8]. Approximately $80 \%$ of the patients improved with imipenem alone and almost the same fraction, $86 \%$, improved with the combination; no statistical difference was detected. It is important, however, to recognise that the weakness of these studies is that the most ill patients were excluded: none of the patients in these studies had an Acute Physiology and Chronic Health Evaluation-II score $>20$. This means that conclusions cannot be drawn regarding the treatment of very sick patients, for whom the results might be different.

Who are the very sick patients? In 1993, ReLlo et al. [12] compiled a list of patients who died during the course of their VAP study. Table 3 indicates that the most common underlying conditions were chronic obstructive pulmonary disease and heart transplantation. Furthermore, almost all the patients had prior antibiotic treatment, which was clearly shown to be a risk factor. At the time of the study, when MRSA was not yet a problem, the leading fatal pathogens were $P$. aeruginosa, Acinetobacter species and Serratia marcescens. These may be the pathogens for which combination therapy should be most seriously considered.

\section{GUIDELINES FOR THE MANAGEMENT OF NOSOCOMIAL PNEUMONIA}

The American Thoracic Society and the Infectious Diseases Society of America have jointly published guidelines for the

\section{TABLE 2 Monotherapy versus combination therapy of hospital-acquired pneumonia}

\begin{tabular}{|c|c|c|c|}
\hline First author [Ref.] & Drugs & Result improvement \% & Comments \\
\hline MANGI [6] & $\begin{array}{l}\text { Cefoperazone versus cefazol/gentamicin or } \\
\text { clindamycin/gentamicin }\end{array}$ & 87 versus 72 & Lower cost for monotherapy \\
\hline Сометта [8] & Imipenem versus imipenem/netilmicin & 80 versus 86 & Combination: 6 nephrotoxic reactions \\
\hline Alvarez Lerma [11] & Meropenem versus ceftazidime/amikacin & 68 versus 55 & Monotherapy better for VAP" \\
\hline
\end{tabular}




\section{TABLE 3 Characteristics of patients who died of ventilator-associated pneumonia}

\begin{tabular}{lccccc} 
Case & Age yrs & Sex M/F & Diagnosis & Prior antibiotic treatment & Microorganisms \\
\hline $\mathbf{1}$ & 43 & M & Heart transplant & Yes & Aspergillus species, Candida species \\
$\mathbf{2}$ & 59 & M & COPD & Yes & P. aeruginosa \\
$\mathbf{3}$ & 33 & M & Heart transplant & Yes & P. aeruginosa, S. marcescens \\
$\mathbf{4}$ & 76 & M & Cranioencephalic trauma & P. aeruginosa \\
$\mathbf{5}$ & 75 & M & Cardiogenic shock & Yes & Aspergillus species \\
$\mathbf{6}$ & 62 & M & CAP & Yes & P. aeruginosa, S. marcescens \\
$\mathbf{7}$ & 70 & M & COPD & Yes & Acinetobacter species, A. calcoaceticus \\
$\mathbf{8}$ & 74 & M & COPD & Yes & P. aeruginosa \\
$\mathbf{9}$ & 71 & F & COPD & Yes & A. calcoaceticus \\
$\mathbf{1 0}$ & 46 & M & Asthma & Pes aeruginosa \\
$\mathbf{1 1}$ & 65 & M & Cardiac surgery & Pes aeruginosa \\
$\mathbf{1 2}$ & 72 & F & Pancreatitis & Yes & P. aeruginosa \\
$\mathbf{1 3}$ & 54 & M & Septic shock & Yes & P. mirabilis \\
$\mathbf{1 4}$ & 21 & M & Multiple trauma & Yes & Uncertain \\
$\mathbf{1 5}$ & 48 & F & Neurosurgery & No & Uncertain \\
$\mathbf{1 6}$ & 68 & M & Cardiac surgery & No & Uncertain \\
$\mathbf{1 7}$ & 51 & M & Multiple trauma & No & S. marcescens \\
$\mathbf{1 8}$ & 71 & M & Thoracic surgery & P. aeruginosa \\
\hline
\end{tabular}

M: male; F: female; COPD: chronic obstructive pulmonary disease; P. aeruginosa: Pseudomonas aeruginosa; S. marcescens: Serratia marcescens; CAP: communityacquired pneumonia; A. calcoaceticus: Acinetobacter calcoaceticus; P. mirabilis: Proteus mirabilis. Reproduced from [12] with permission from the publisher.

treatment of hospital-acquired pneumonia, including VAP, along with certain other pneumonia cases that may be viewed as associated with the use of healthcare facilities [13]. These may be considered international guidelines, since expert physicians from Europe and Argentina were also involved in their development.

As a general framework for the choice between monotherapy and combination therapy, the guidelines indicate that

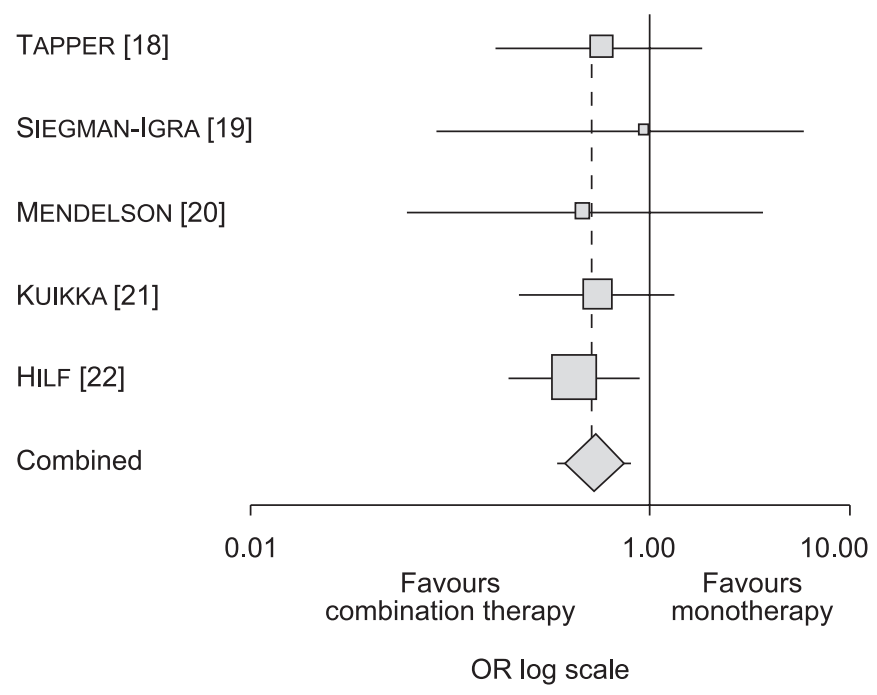

FIGURE 4. Analysis of studies comparing the effect of combination antiinfective therapy versus monotherapy on mortality in Pseudomonas aeruginosa bacteraemia. 1 : odds ratio $(\mathrm{OR})$ with size denoting the proportion of information given by each trial; — : 95\% confidence interval. Reproduced from [17] with permission from the publisher.
"Monotherapy should be used when possible because combination therapy is often expensive and exposes patients to unnecessary antibiotics, thereby increasing the risk of multidrug-resistant (MDR) pathogens and adverse outcomes" [13]. Contrary to widespread belief, combination therapy does not generally significantly reduce the likelihood that resistance will appear [14].

When is combination therapy necessary? The guidelines draw a clear distinction between situations where there are risk factors for MDR pathogens and those where risk factors are absent. The most common risk factor is onset after $\geqslant 5$ days of hospitalisation. However, even those with early-onset pneumonia may be considered at high risk if: 1) they have had antimicrobial therapy within the preceding 90 days; 2) if there is a high frequency of resistant pathogens in the community or specific hospital unit; 3) if a family member has a multidrugresistant infection; or 4) if they have had certain types of contact with healthcare facilities, such as hospitalisation for at least 2 of the preceding 90 days, nursing home residence, chronic dialysis or home wound care. The immunosuppressed patient is also considered to be at high risk [13].

A significant change from the previous guidelines is that the distinction between early and late onset is now based on the time from admission to the hospital rather than the time from admission to the ICU.

The therapeutic recommendations do not depend in any way on the severity of the disease, but rather on the likelihood that the pathogen will exhibit resistance. If there are no risk factors, then the disease is assumed to be due to one of the antibioticsensitive pathogens common in the community. The recommendation is for monotherapy with a limited-spectrum 
antibiotic, such as ceftriaxone, levofloxacin, moxifloxacin, ampicillin/sulbactam or ertapenem [14].

If there are risk factors for MDR pathogens, then the guidelines call for broad-spectrum therapy and the early use of combination therapy. A major rationale is that the combined spectrum of two antibiotics is broader than that of one, thus increasing the chances that at least one will be active against a pathogen that is still unidentified [13]. The combination chosen should include drugs from different classes, with one drug being an antipseudomonal cephalosporin, an antipseudomonal carbapenem or a $\beta$-lactam/ $\beta$-lactamase inhibitor, while the other should be either an antipseudomonal fluoroquinolone or an aminoglycoside. Additionally, if MRSA is a plausible pathogen, either vancomycin or linezolid should be added (triple therapy) [13]. The guidelines note that a carbapenem is the preferred $\beta$-lactam if Acinetobacter species or ESBL-producing K. pneumoniae are suspected, while a macrolide or a fluoroquinolone rather than an aminoglycoside should be used if there is suspicion of Legionella pneumophila [13].

Agents for use in broad-spectrum therapy should have a low potential to select for resistance, as well as potent activity against $P$. aeruginosa. Examples among the $\beta$-lactams include cefepime and meropenem, which are currently approved, and doripenem, which has recently completed phase III trials [15, 16]. Ceftazidime and imipenem, however, do not meet these criteria.

Once the specific pathogen is identified, therapy should be deescalated to an antibiotic specific for that pathogen, generally implying monotherapy [13].

\section{CONTROVERSIES AND CONCLUSIONS}

The advantages of combination therapy have not been well documented, although it is generally agreed that the single organism most likely to call for combination therapy is $P$. aeruginosa. Unfortunately, comparative clinical data for nosocomial pneumonia or VAP due to $P$. aeruginosa appear to be lacking, and such studies are badly needed. A meta-analysis of studies comparing combination therapy with monotherapy in another serious disease, Gram-negative bacteraemia, found 17 studies that reported both monotherapy and combination therapy and data on mortality [17]. Most studies used $\beta$-lactams or aminoglycosides alone or in combination. The summary odds ratio was 0.96 (95\% confidence interval 0.70 1.32), indicating that there was no benefit in mortality with combination therapy. However, a subgroup analysis of only $P$. aeruginosa bacteraemia showed a significant benefit to mortality (fig. 4). Although the results for only one of the individual studies reached statistical significance, the combined analysis did show a statistically significant advantage for combination therapy. Therefore, routine use of combination therapy for serious Gram-negative infections may not provide a death benefit, but use of combination therapy against $P$. aeruginosa may reduce mortality [17].

More than a decade ago, it was thought that combination therapy should be used for difficult-to-treat Gram-negative bacteria, including Pseudomonas aeruginosa, Acinetobacter species, and Enterobacteriaceae producing extended-spectrum $\beta$-lactamases. Combination therapy would likely produce a synergistic antibiotic effect, prevent the emergence of resistance and extend the spectrum of antibacterial activity [23]. However, the clinical literature since the late 1980s does not provide concrete support for any of these theoretical arguments. Nevertheless, it must be kept in mind that all the published trials exclude the most severe cases and, therefore, may not reflect the full potential success of combination therapy in the treatment of the more serious cases of ventilator-associated pneumonia.

\section{REFERENCES}

1 Jones ME, Draghi DC, Thornsberry C, Karlowsky JA, Sahm DF, Wenzel RP. Emerging resistance among bacterial pathogens in the intensive care unit-a European and North American Surveillance study (2000-2002). Ann Clin Microbiol Antimicrob 2004; 3: 14.

2 Jones ME, Karlowsky JA, Draghi DC, Thornsberry C, Sahm DF, Nathwani D. Epidemiology and antibiotic susceptibility of bacteria causing skin and soft tissue infections in the USA and Europe: a guide to appropriate antimicrobial therapy. Int J Antimicrob Agents 2003; 22: 406-419.

3 Eggimann P, Revelly JP. Should antibiotic combinations be used to treat ventilator-associated pneumonia? Semin Respir Crit Care Med 2006; 27: 68-81.

4 Mouton JW, van Ogtrop ML, Andes D, Craig WA. Use of pharmacodynamic indices to predict efficacy of combination therapy in vivo. Antimicrob Agents Chemother 1999; 43: 2473-2478.

5 Damas P, Garweg C, Monchi M, et al. Combination therapy versus monotherapy: a randomised pilot study on the evolution of inflammatory parameters after ventilatorassociated pneumonia [ISRCTN31976779]. Crit Care 2006; 10: R52.

6 Mangi RJ, Greco T, Ryan J, Thornton G, Andriole VT. Cefoperazone versus combination antibiotic therapy of hospital-acquired pneumonia. Am J Med 1988; 84: 68-74. Erratum in: Am J Med 1988; 84: 800.

7 Fink MP, Snydman DR, Niederman MS, et al. Treatment of severe pneumonia in hospitalized patients: results of a multicenter, randomized, double-blind trial comparing intravenous ciprofloxacin with imipenem-cilastatin. The Severe Pneumonia Study Group. Antimicrob Agents Chemother 1994; 38: 547-557.

8 Cometta A, Baumgartner JD, Lew D, et al. Prospective randomized comparison of imipenem monotherapy with imipenem plus netilmicin for treatment of severe infections in nonneutropenic patients. Antimicrob Agents Chemother 1994; 38: 1309-1313.

9 Rubinstein E, Lode H, Grassi C. Ceftazidime monotherapy versus ceftriaxone/tobramycin for serious hospitalacquired gram-negative infections. Antibiotic Study Group. Clin Infect Dis 1995; 20: 1217-1228.

10 Sieger B, Berman SJ, Geckler RW, Farkas SA. Empiric treatment of hospital-acquired lower respiratory tract infections with meropenem or ceftazidime with tobramycin: a randomized study. Meropenem Lower Respiratory Infection Group. Crit Care Med 1997; 25: 1663-1670.

11 Alvarez Lerma F; Serious Infection Study Group. Efficacy of meropenem as monotherapy in the treatment of ventilator-associated pneumonia. J Chemother 2001; 13: 70-81. 
12 Rello J, Ausina V, Ricart M, Castella J, Prats G. Impact of previous antimicrobial therapy on the etiology and outcome of ventilator-associated pneumonia. Chest 1993; 104: 1230-1235.

13 American Thoracic Society; Infectious Diseases Society of America. Guidelines for the management of adults with hospital-acquired, ventilator-associated, and healthcareassociated pneumonia. Am J Respir Crit Care Med 2005; 171: 388-416.

14 Cunha BA. Ventilator-associated pneumonia: monotherapy is optimal if chosen wisely. Crit Care 2006; 10: 141.

15 Mushtaq S, Ge Y, Livermore DM. Doripenem versus Pseudomonas aeruginosa in vitro: activity against characterized isolates, mutants, and transconjugants and resistance selection potential. Antimicrob Agents Chemother 2004; 48: 3086-3092.

16 Sakyo S, Tomita H, Tanimoto K, Fujimoto S, Ike Y. Potency of carbapenems for the prevention of carbapenem-resistant mutants of Pseudomonas aeruginosa: the high potency of a new carbapenem doripenem. J Antibiot (Tokyo) 2006; 59: 220-228.

17 Safdar N, Handelsman J, Maki DG. Does combination antimicrobial therapy reduce mortality in Gram-negative bacteraemia? A meta-analysis. Lancet Infect Dis 2004; 4: 519-527.

18 Tapper ML, Armstrong D. Bacteremia due to Pseudomonas aeruginosa complicating neoplastic disease: a progress report. J Infect Dis 1974; 130: S14-S23.

19 Siegman-Igra Y, Ravona R, Primerman H, Giladi M. Pseudomonas aeruginosa bacteremia: an analysis of 123 episodes, with particular emphasis on the effect of antibiotic therapy. Int J Infect Dis 1998; 2: 211-215.

20 Mendelson MH, Gurtman A, Szabo S, et al. Pseudomonas aeruginosa bacteremia in patients with AIDS. Clin Infect Dis 1994; 18: 886-895.

21 Kuikka A, Valtonen VV. Factors associated with improved outcome of Pseudomonas aeruginosa bacteremia in a Finnish university hospital. Eur J Clin Microbiol Infect Dis 1998; 17: 701-708.

22 Hilf M, Yu VL, Sharp J, Zuravleff JJ, Korvick JA, Muder RR. Antibiotic therapy for Pseudomonas aeruginosa bacteremia: outcome correlations in a prospective study of 200 patients. Am J Med 1989; 87: 540-546.

23 Bergogne-Berezin E. Treatment and prevention of nosocomial pneumonia. Chest 1995; 108: Suppl. 2, 26S-34S. 